\title{
Proposta conceitual de estudo para a integração e gerenciamento do uso de marcadores bioquímicos de contaminação ambiental
}

\author{
Vera Lúcia S. S. de Castro* \\ Cláudio Martin Jonsson \\ Laboratório de Ecotoxicologia e Biossegurança, Embrapa Meio Ambiente \\ Rodovia SP 340, km 127,5, CEP 13820-000, Jaguariúna - SP, Brasil \\ *Autor para correspondência \\ castro@cnpma.embrapa.br
}

Submetido em 10/04/2011

Aceito para publicação em 25/11/2011

\section{Resumo}

A agricultura sustentável prioriza o uso racional da terra e da biota. Para tanto, é necessária a compreensão da interação entre os insumos agrícolas e os ecossistemas. Porém, há carência de métodos de fácil acesso para a verificação de contaminação ambiental por muitos desses produtos, como os agrotóxicos e de suas misturas. A aplicação de métodos alternativos no monitoramento de áreas degradadas pode gerar vantagens econômicas quando comparados à implementação de protocolos de análise que utilizam métodos de alto custo que demandam maior tempo de análise. Uma vez que a saúde ambiental pode ser avaliada por meio de estudos com indicadores biológicos, a abordagem aqui apresentada objetiva discutir alguns aspectos a respeito do monitoramento de risco ambiental com organismos não-alvo sentinela, focando parâmetros bioquímicos. Tais parâmetros evidenciariam que o agrotóxico penetrou no organismo e ocasionou uma resposta a um efeito de acordo com a dose ou o tempo de exposição. Esses parâmetros poderão ser utilizados como indicadores biológicos bioquímicos em programas de monitoramento ambiental devido à identificação precoce de alterações. Os resultados obtidos de seu uso poderão ser utilizados para subsidiar políticas relacionadas ao gerenciamento de riscos ambientais.

Palavras-chave: Agrotóxicos, Árvore de decisão, Enzimas, Indicadores biológicos, Monitoramento ambiental

\section{Abstract}

Conceptual study proposal for the integration and management of biochemical markers of environmental contamination use. Sustainable agriculture prioritizes the rational use of land and biota. For this, there is a need for understanding the interaction between the agriculture inputs and the ecosystems. However, there is a lack of easy access methods for verifying environmental contamination due to many of these products, such as pesticides and their mixtures. The application of alternative methods for monitoring degraded areas can generate economic advantages when compared to the implementation of protocols for analysis using high cost methods which demand a longer analysis time. Since environmental health can be accessed through studies using biomarkers, the approach presented here aims to discuss some aspects on the environmental risk monitoring with sentinel non-target organisms, focusing on biochemical parameters. Such parameters showed that the pesticide penetrated into the body and caused a response according to the dose or time of exposition. 
These parameters shall be used as biochemical biomarkers in programs for environmental monitoring because of the early identification of changes. The results obtained through its use shall be used to support policies related to environmental risks management.

Key words: Biomarker, Decision tree, Environmental monitoring, Enzymes, Pesticides

\section{Introdução}

A contaminação ambiental originada por agrotóxicos, que pode decorrer de sistemas intensivos agrícolas, pode causar efeitos negativos aos recursos naturais, à saúde humana, além de trazer problemas para a exploração agrícola. Este fato leva à preocupação quanto ao seu impacto nas populações e na qualidade ambiental (ARAÚJO et al., 2007; ECKERMAN et al., 2007). Os riscos devido às perturbações ambientais são de difícil predição devido à complexidade das interações e variabilidade dos ecossistemas e seus organismos, sendo necessária a compreensão da interação entre os insumos agrícolas como os agrotóxicos e os ecossistemas para assegurar o uso sustentável destes compostos no ambiente.

Nesse contexto, a aplicação de métodos alternativos de monitoramento dos efeitos negativos dos insumos externos em áreas afetadas pode gerar vantagens econômicas quando comparados à aplicação de protocolos tradicionais de análise que utilizam reagentes e equipamentos de alto custo e de maior tempo de análise. Os métodos analíticos tradicionais capazes de avaliar agrotóxicos em água são realizados por técnicas cromatográficas como cromatografia gasosa (CG), CLAE (cromatografia líquida de alta eficiência) ou espectotrometria de massa. No entanto, esses métodos de análise exigem mão-de-obra especializada e apresentam alto custo operacional, o que torna inviável sua aplicação em larga escala. Além disso, a poluição ambiental associada ao uso de pesticidas é especialmente difícil de ser avaliada, pois os eventos de contaminação obedecem a uma dinâmica espacialmente difusa e temporalmente variável (ARIAS et al., 2007).

As misturas de agrotóxicos podem decorrer do uso concomitante de diferentes ingredientes ativos em formulações comerciais e em decorrência das respectivas persistências nos compartimentos ambientais. De fato, apesar de misturas em tanques estarem proibidas por lei; a ocorrência cada vez mais precoce de doenças fúngicas nas lavouras, atingindo diferentes estádios vegetativos das plantas, tem suscitado o uso de misturas de fungicidas que tenham apresentação comercial para várias culturas, e que contenham ingredientes ativos como grupos triazóis (ciproconazol, epoxiconazol e tebuconazol) e derivados de estrobilurinas (BARROS et al., 2005; REIS FILHO et al., 2007).

A identificação dos fatores que levam à ocorrência de efeitos toxicológicos significativos devido à exposição de organismos às misturas de agrotóxicos deverá fornecer um retrato mais detalhado antes da realização de avaliações de risco que possam ser aplicadas a misturas (ARNOLD; PRICE, 2007). Nesse sentido, é importante a determinação de concentrações de misturas que causem efeitos não desejáveis em espécies não-alvo (CORYSLECHTA, 2005; NEEDHAM et al., 2005; COSTA et al., 2009) (Concentração de Efeito Não Observado - CENO).

A toxicidade das misturas de agrotóxicos ou outros compostos nem sempre é resultante da soma das atividades tóxicas dos compostos (JIN-CLARK et al., 2002; DENTON et al., 2003). Assim, os agrotóxicos podem ter ação conjunta capaz de provocar efeitos tóxicos maiores ou menores nos organismos do que seria esperado a partir do conhecimento sobre os compostos isolados. Um dos pontos principais a considerar é se haverá ou não interação e caso houver se ela ocorrerá na forma de um sinergismo ou antagonismo (POHL; ABADIN, 2008; REFFSTRUP et al., 2010). Possíveis interações entre os compostos podem ocorrer na fase cinética (absorção, distribuição, metabolização e excreção) ou na fase dinâmica (efeitos do composto no receptor, alvo celular, etc.) (GROTEN et al., 2004).

De forma geral, os modelos usados atualmente para o estudo do efeito de misturas de agrotóxicos se 
baseiam na análise da resposta do organismo a diferentes doses de exposição e lançam mão de abordagens estatísticas de estimação (ANDERSEN; DENNISON, 2004; CORY-SLECHTA, 2005; EL-MASRI, 2007). Várias abordagens têm sido sugeridas nos estudos científicos publicados em relação à avaliação dos riscos à saúde decorrentes da exposição a misturas de produtos químicos, embora não exista um procedimento internacionalmente aceito (POUNDS et al., 2004; ELMASRI, 2007; ANDERSEN et al., 2009; BALDWIN; ROLING, 2009; REFFSTRUP et al., 2010). A escolha do método para o estudo da interação depende do efeito tóxico, dos dados disponíveis a respeito da toxicidade da mistura ou de seus compostos além dos dados a respeito da exposição aos compostos.

Em geral, na avaliação do risco de misturas, é identificado um grupo de compostos para estudo que possuem estrutura química semelhante ou induzem um determinado efeito por um mecanismo comum de toxicidade como a atuação em um mesmo alvo molecular ou a formação de um intermediário tóxico em comum (USEPA, 2002). Contudo, o foco da pesquisa em um grupo de compostos com a mesma estrutura ou o mesmo mecanismo de ação tem sido questionado por alguns autores (CORY-SLECHTA, 2005; CEDERGREEN et al., 2008).

Alguns métodos podem ser utilizados para as misturas de compostos que são toxicologicamente semelhantes: o método do índice de risco (hazard index method - HI) (GUTIÉRREZ et al., 2008), o método do fator de potência relativa (relative potency factor method - RPF) (SMIALOWICZ et al., 2008) e o método do fator de equivalência de toxicidade (toxicity equivalency factor method - TEF) (TOYOSHIBA et al., 2004).

Em ecotoxicologia, há uma preocupação crescente a respeito da falta de precisão dos métodos atualmente empregados e da inclusão destes estudos na avaliação da qualidade da água (TEUSCHLER, 2007; BELLAS, 2008; CHÈVRE et al., 2008; GUTIÉRREZ et al., 2008). A aplicação de algumas metodologias como o cálculo da concentração fracionária inibitória (Fractional Inhibitory Concentration - FIC) (LIRA et al., 2001) permite estudar e classificar o tipo de interação entre os poluentes. Contudo, alguns problemas dificultam a avaliação dos possíveis efeitos que as misturas possam produzir como o pequeno conhecimento relativo sobre a magnitude, duração, frequência e tempo de exposição cumulativa ambiental, bem como a compreensão insuficiente sobre os mecanismos interativos de toxicidade entre os componentes da mistura. Nesse sentido, há poucas evidências disponíveis sobre a mistura e seus efeitos relacionados em níveis de exposição reais (MASON et al., 2007).

Nos últimos anos, modelos matemáticos que levam em consideração parâmetros associados à estrutura química dos compostos da mistura têm sido aperfeiçoados a fim de predizer a interação esperada. Assim, estudos sobre a relação quantitativa da estrutura-atividade (Quantitative Structure-Activity Relationship - QSAR), onde as estruturas moleculares são correlacionadas a efeitos biológicos conhecidos, fazem parte de uma estratégia para diagnosticar a ação conjunta de agentes químicos ainda não experimentados in vivo ou para suportar a escolha e priorização dos compostos que deverão ser submetidos a novos testes toxicológicos (PERY et al., 2010).

Alguns modelos foram propostos considerando propriedades da molécula como o coeficiente de partição octanol/água (Kow) (BAAS et al., 2009; LU et al., 2009) e parâmetros moleculares como volume molar (Vm) entre outros (ZHANG et al., 2007; WANG et al., 2008) além da presença de produtos resultantes de processo de biotransformação, quando estes podem produzir efeitos adversos e contribuir para o risco estimado total (NEUWOEHNER et al., 2010).

Desta forma, verifica-se que a avaliação de riscos cumulativos depende do desenvolvimento de métodos e procedimentos que relacionem os dados de exposição aos de efeito através de indicadores a fim de caracterizar os riscos a saúde ambiental com um grau aceitável de precisão (SEXTON; HATTIS, 2007). Assim, o objetivo do presente trabalho é formular uma proposta conceitual para o estudo das respostas dos indicadores bioquímicos decorrentes da exposição de organismos a misturas de agrotóxicos no ambiente. A proposta visa à integração e ao gerenciamento dos dados obtidos com o uso destes indicadores. 
A seguir, são abordadas questões relativas à importância do estudo e do uso de indicadores biológicos para a avaliação da toxicidade na área de estudo, e são apresentadas as fases sequenciais da proposta elaborada.

\section{Indicadores biológicos}

Alguns autores distinguem os conceitos de biomarcador e bioindicador. Segundo Nascimento et al. (2006), os biomarcadores são definidos como respostas biológicas adaptativas a agentes estressores, evidenciadas como alterações bioquímicas, celulares, histológicas, fisiológicas ou comportamentais. O biomarcador compreende então toda substância ou seu produto de biotransformação ou qualquer alteração bioquímica precoce cuja determinação nos fluidos biológicos, tecidos ou ar exalado estabeleça a relação entre a exposição e o efeito tóxico refletida em alterações pré-clínicas e agravos à saúde ou ainda através do entendimento dos mecanismos relacionados à ação tóxica e o grau de resposta nos indivíduos (AMORIM, 2003). Por sua vez, os bioindicadores são considerados como organismos ou comunidades que respondem à poluição ambiental, alterando suas funções vitais e/ ou sua composição química e com isso tornam-se ferramentas úteis na avaliação da situação ambiental de uma dada área (WU et al., 2010).

A sensibilidade de um organismo a um poluente pode variar. Um nível seguro para uma espécie de peixe pode não o ser para outra espécie que faz parte da mesma comunidade biológica. A sensibilidade de um organismo frente à qualidade de seu ambiente depende não somente do fator a ser monitorado, mas também do nível nutricional, idade do organismo, sexo, fase de desenvolvimento, características genéticas, competição entre indivíduos ou espécies, etc., além de fatores ambientais como luminosidade e temperatura. Assim, por exemplo, para a detecção de despejos tóxicos no ambiente, as amostras devem ser coletadas em vários pontos localizados a montante do ponto de despejo, preferencialmente além da área de mistura e a jusante do ponto de descarte. Cuidados devem ser tomados para incluir uma área controle e uma área de recuperação, assim como várias estações intermediárias respeitando o gradiente de poluição. A padronização dos organismos bioindicadores é por isso um fator de fundamental importância em programas de monitoramento. Para a escolha do organismo-teste geralmente usam-se os seguintes critérios de seleção de espécies: abundância e disponibilidade; significativa representação ecológica dentro das biocenoses; cosmopolitismo da espécie; conhecimento da sua biologia, fisiologia e hábitos alimentares; estabilidade genética e uniformidade de suas populações; baixo índice de sazonalidade; sensibilidade constante e apurada; importância comercial; facilidade de cultivo em laboratório e, se possível, a espécie deve ser nativa para a melhor representatividade dos ecossistemas. Considerando-se a dificuldade em encontrar uma espécie com todas estas características, existem muitas espécies padronizadas que podem ser utilizadas, sendo extremamente importante a realização de bioensaios com espécies representativas do ambiente de estudo, pois respondem melhor as condições encontradas nos ambientes naturais onde vivem. Várias espécies vêm sendo empregadas internacionalmente em testes de toxicidade em ensaios laboratoriais, sendo que dentre os principais grupos de organismos destacam-se: microalgas, microcrustáceos, poliquetas, oligoquetas, peixes e bactérias, representando os mais diversos ecossistemas e níveis tróficos. No entanto, não existe uma única espécie de organismo que represente integralmente os efeitos causados em um determinado ecossistema. É necessário, portanto, desenvolver estudos no sentido de investigar o potencial de uso de espécies nativas no biomonitoramento ecotoxicológico (MAGALHÃES; FERRÃO, 2008).

Outros autores por sua vez compreendem que ao nível de organização biológica do organismo, as ferramentas de análise são chamadas de bioindicadores. Bioindicadores são então definidos como qualquer resposta a um contaminante ambiental ao nível individual, medidos no organismo ou matriz biológica, indicando um desvio do status normal que não pode ser detectado no organismo intacto. Ou seja, são medidas de fluidos corporais, células, tecidos ou medidas realizadas sobre o organismo completo que indicam, em termos bioquímicos, celulares, fisiológicos, compartimentais ou energéticos, a presença de substâncias contaminantes ou a magnitude da resposta do organismo alvo. Um indicador biológico pode ser então entendido como 
uma mudança na resposta biológica variando desde uma alteração molecular, celular ou comportamental, que possa ser relacionada à exposição a contaminantes como o agrotóxico e a uma resposta do organismo indicando um desvio de seus parâmetros normais em condições habituais (ARIAS et al., 2007). Estes efeitos podem preceder outros e servem como alerta para a ocorrência de efeitos em longo prazo (LAMA; GRAY, 2003).

Apesar dos efeitos biológicos e bioquímicos poderem vincular a biodisponibilidade dos compostos de interesse com a sua concentração em órgãos-alvo e toxicidade intrínseca, as limitações do biomonitoramento, como os fatores que não estão relacionados com a poluição; devem ser cuidadosamente considerados na interpretação dos dados (VAN DER OOST et al., 2003) já que algumas características biológicas geralmente respondem a diferentes estressores e, portanto não são susceptíveis de discriminar os efeitos de múltiplos estressores (LIESS et al., 2008). Para tanto, é necessário conhecer as condições de exposição no ambiente e melhorar a compreensão da toxicocinética da mistura total e seus componentes, além de características das espécies em estudo (MUMTAZ et al., 2007; RICE et al., 2008; GOMEZ-EYLES et al., 2009). Apesar disso, em uma investigação inicial de monitoramento quando é necessário estabelecer os pontos onde será feito um estudo mais criterioso; pode-se utilizar uma abordagem generalista, mas útil como a indução de proteínas de estresse como heat shock proteins (HSP) que incluem um grande número de isoformas (e.g. - HSP70) e que representam resposta não específica à poluição (NASCIMENTO et al., 2006).

\section{Propostadeabordagemconceitual para o estudo de efeitos ecotoxicológicos decorrentes de misturas}

Alguns ecossistemas podem apresentar-se difusamente poluídos por misturas de contaminantes. Esta situação não tem consequências claras, especialmente quando os gestores não possuirem meios de determinar se as condições encontradas nos sistemas de água podem ser consideradas ecologicamente adequadas. Como então identificar com praticidade e precisão, a existência de riscos ao meio ambiente de forma a facilitar a interpretação dos problemas para uma tomada de decisão efetiva e eficaz no processo de avaliação do risco de intoxicação ambiental das misturas de agrotóxicos?

A integração de dados passíveis de serem coletados em diferentes aspectos como a composição da mistura de compostos e a sensibilidade das espécies locais podem subsidiar as ações de proteção às populações de diferentes organismos expostas aos agrotóxicos. As informações referentes a um determinado sítio podem ser usadas em conjunto com outras baseadas em conhecimento de ecologia a fim de avaliar e em consequência promover a gestão da poluição que se apresenta de forma difusa no ambiente (POSTHUMA et al., 2008). Alguns trabalhos propõem estudos em fases sequenciais na dependência dos dados disponíveis, nas características dos compostos estudados e dos métodos disponíveis (TEUSCHLER, 2007; WHO, 2009; REFFSTRUP et al., 2010). Nesse sentido, é interessante testar inicialmente as possíveis misturas em laboratório e posteriormente em estudos de biomonitoramento que podem ocorrer em diferentes ambientes (MATTSSON, 2007). Com os dados de biomonitoramento será possível estabelecer e verificar as relações entre a exposição aos compostos e a ocorrência de mudanças rápidas nas respostas dos marcadores bioquímicos que funcionariam como marcadores preditivos de curto prazo para efeitos adversos em longo prazo (VAN DER OOST et al., 2003).

Uma vez que atualmente, o objetivo dos estudos relacionados à avaliação dos impactos em áreas agriculturáveis é o desenvolvimento e aperfeiçoamento de metodologias e ferramentas que permitam determinar de forma realista a condição ambiental (ARIAS et al., 2007); este trabalho propõe uma abordagem em fases sequenciais de avaliação de locais potencialmente contaminados por misturas de agrotóxicos por meio de indicadores biológicos, a qual pode contribuir para aprimorar o gerenciamento e a implementação de políticas e decisões gerenciais para os ecossistemas. Na sequência, são apresentadas as fases do estudo. 


\section{Fase I - Estabelecimento do padrão qualitativo e quantitativo do indicador enzimático frente a agrotóxicos em comparação a resposta dos organismos sentinela em condições não poluídas de água}

Na avaliação das alterações de um ecossistema, fazse necessário o conhecimento das oscilações em situação de normalidade dos parâmetros a serem analisados em organismos que representam os alvos dos mecanismos de toxicidade. Assim, é necessário padronizar inicialmente os parâmetros normais de atividade enzimática de vários organismos sentinela em diferentes níveis tróficos e posteriormente mensurar respostas biológicas através de curvas dose-resposta em ensaios laboratoriais voltadas à definição de concentração para os efeitos observados.

Nesta fase são identificadas e definidas as enzimas de interesse nos organismos sentinela. Idealmente, as enzimas selecionadas como indicadores ambientais (VAN DER OOST et al., 2003) devem possuir as seguintes características: quantificação facilmente realizável com qualidade e o menor custo possível, resposta sensível à exposição ao agrotóxico, possuir padrão de comportamento definido referente à variabilidade natural dentro da espécie avaliada em um determinado nível trófico, tempo e dose de exposição ao poluente bem como possuir significância toxicológica entre a sua resposta e o impacto no organismo. Dados de literatura mostram a importância do uso de multiindicadores em peixes na avaliação do impacto da poluição em rios (LINDE-ARIAS et al., 2008).

As enzimas a serem estudadas serão definidas inicialmente com base em dados bibliográficos e testes preliminares da atividade enzimática frente a vários produtos elencados com base na frequência de uso e toxicidade. Assim, são citadas as enzimas envolvidas no estresse oxidativo que vem sendo bastante estudadas (LUSHCHAK, 2011). A alteração da atividade das enzimas em testes in vitro ou extraídas de organismos expostos a poluentes químicos de diversas origens, tem sido alvo de estudo na análise de risco e monitoramento de áreas degradadas (REISFELD; YANG, 2004; WANG; FOWLER, 2008) já que as respostas moleculares e bioquímicas ocorrem rapidamente (ARIAS et al., 2007).
Os estudos dos marcadores bioquímicos podem então fornecer informações úteis sobre os mecanismos de ação e os efeitos decorrentes da interação entre os organismos e as misturas de agrotóxicos presentes no ambiente, que são de grande valor para as práticas de avaliação de risco (WANG; FOWLER, 2008).

Uma grande importância tem sido dada aos efeitos sobre enzimas envolvidas no processo de biotransformação, tanto em sua fase I como na fase II. A fase I compreende um conjunto de reações de oxidação, redução e hidrólise. Essas reações, geralmente, modificam a estrutura química da substância resultando em aumento de hidrofilicidade. As reações de biotransformação de fase II, também chamadas de reações de conjugação, incluem glicuronidação, sulfonação, acetilação, metilação, conjugação com glutationa e com aminoácidos. Na maioria das reações de conjugação formam-se compostos altamente polarizados e hidrossolúveis que são excretados pelos rins.

Entre as primeiras, associadas às atividades do citocromo P450 e de monoxigenases de função mista, têm sido avaliadas a etoxiresorufina-o-de-etilase (EROD) e benzopireno monoxigenases (FENET et al., 1998; RAMLJAK et al., 2000; RUUS et al., 2002). Com relação às enzimas de conjugação na fase II, a glutationaS-transferase (GST) e a UDP-glucuronosiltransferase (UDPGT) têm sido também bastante estudadas em peixes expostos em condições de laboratório e naqueles coletados de áreas impactadas (TAYSSE et al. 1998; JONSSON et al., 2002; TARJA et al., 2003).

Apesar dos estudos referentes à fase I serem abundantes, ainda são menos numerosos os estudos a respeito da interferência quanto a possível interação de misturas de compostos na fase II (ANAND; MEHENDALE, 2004). Também as inibições das atividades da acetilcolinesterase e delta-aminolevulinode-hidratase vêm sendo investigadas em peixes e outros organismos (JIN-CLARK et al., 2002; DENTON et al., 2003). A seleção do indicador biológico deverá também considerar diversas questões como a existência de regulamentações, a escala de paisagem, os produtos recomendados e os utilizados (doses, método e frequência de aplicação). 
Fase II - Padronização e validação da metodologia para a quantificação dos indicadores enzimáticos selecionados visando o entendimento de sua relação exposição-resposta

Neste item, são estabelecidas as características para a verificação da adequação dos indicadores enzimáticos selecionados no que diz respeito à especificidade $\mathrm{e}$ ao significado das alterações observadas e a revisão dos procedimentos analíticos para a quantificação do indicador e suas limitações. Para tanto, os indicadores devem ter seu método de quantificação validado. No caso de indicadores enzimáticos, é construída a curva padrão para estudar a relação entre a exposição a diferentes concentrações do agrotóxico e são estabelecidos os parâmetros experimentais e a otimização do método com a quantificação de limites. Devem ser estabelecidos limites de indução/inibição da atividade dos biomarcadores bioquímicos (enzimas) em organismos sentinela de compartimentos de água com alta correlação com os níveis de poluição observados.

\section{Fase III-Biomonitoramento dos indicadores a fim de detectar a extensão e a natureza da exposição a que o organismo está exposto}

Nesta Fase é estudada a variação de resposta entre as populações de organismos expostas e não-expostas aos agentes estressores em estudos de biomonitoramento considerando as possíveis interferências no meio ambiente. O biomonitoramento consiste em uma apreciação integrada de diferentes respostas induzidas pelo estresse do organismo, que tenta, em conjunto com outros parâmetros ambientais, produzir um retrato da situação de poluição visando à proteção da saúde ecológica. Porém, os dados que incluem os efeitos observados no campo, devem considerar fatores relacionados ao ambiente e aos organismos em estudo (ARNOT et al., 2011) já que nem sempre podem ser vinculados a um estressor específico e o conhecimento dos efeitos pelo stress na biota desencadeado pelos contaminantes muitas vezes ainda é insuficiente (SIONTOROU; BATZIAS, 2011).
Em vista disso, as observações e dados obtidos podem ser efetivamente transformados em conhecimento em três passos: (i) as observações e medições, verificadas de acordo com normas aprovadas, transformam-se em dados, ii) os dados, devidamente selecionados, testados e relacionados às áreas em estudo tornam-se informação; (iii) as informações, organizadas e interpretadas ou aplicadas às áreas de interesse, se tornam conhecimento.

O conhecimento, se assimilado, apreciado e enriquecido de modo que seja compreendido, aferido e integrado leva ao entendimento. Por sua vez, este conhecimento colocado em perspectiva e analisado sob certos valores leva às políticas ambientais eficientes e relevantes que perdem o seu caráter local e se tornam uma referência ou um padrão para casos análogos. As ferramentas de gerenciamento são consequentemente indispensáveis para transformar os resultados do biomonitoramento em avaliação de risco (SIONTOROU; BATZIAS, 2011).

\section{Fase IV - Gerenciamento da informação obtida}

Uma vez que os perigos foram identificados deve-se estabelecer se há necessidade de controle e que medidas são necessárias para cada ponto de controle. O critério para aceitabilidade de risco vai depender das características de cada caso em questão e deve ser aberto a constantes revisões a fim de permitir a incorporação de novos achados.

Alguns obstáculos ainda precisam ser superados antes que os métodos enzimáticos sejam utilizados em uma base ampla, mas estes métodos podem permitir uma avaliação mais rápida da problemática da contaminação ambiental. Assim, por exemplo, deve ser ainda discutida a forma como o limite de detecção atingido pelas avaliações enzimáticas se correlaciona com os limites aceitáveis da legislação em vigor (VAN DYK; PLETSCHKE, 2011).

Por sua vez, a aceitabilidade do risco não é um conceito universal. Há dificuldade na determinação do valor do risco aceitável e na definição das premissas e conceitos associados que acabam por resultar no debate de questões éticas, sociais, políticas e culturais que afetam o julgamento do risco (GREIG-SMITH, 
1992). Além destes, há aqueles fatores inerentes ao método de avaliação de risco como, por exemplo, o estabelecimento do peso de cada evidência que pode ser um teste laboratorial, múltiplas estimativas de um parâmetro ou um estudo a campo.

Dentro de cada nível de interação entre os compostos, desde aqueles envolvidos nos processos da exposição ambiental, da toxicocinética e da toxicodinâmica; podem ser usadas avaliações experimentais além de abordagens computacionais para investigar os efeitos de uma mistura de compostos. O papel fundamental de importantes enzimas de desintoxicação, como a família citocromo P450 (CYPs), glutationa-S-transferases, e metaloproteínas, tornam as mesmas candidatas a estes estudos (SPURGEON et al., 2010).

$\mathrm{Na}$ construção de um modelo que envolve múltiplas hipóteses, a abordagem mais útil é criar um modelo conceitual que combine os dados selecionados individuais a priori em uma nova estrutura causal (SUTER II; CORMIER, 2011). Todavia, uma vez que a cadeia de informação depende da consistência entre as pesquisas, a comparabilidade dos dados e a sua acessibilidade devem ser melhoradas para gerar informações mais abrangentes sobre a dinâmica espacial e temporal da exposição. Isso requer algum tipo de normalização/harmonização que reforçaria as correlações observadas entre os efeitos da exposição e da saúde, que em última instância pode apontar para possíveis relações causais (SIONTOROU; BATZIAS, 2011).

Atualmente, a capacidade dos sistemas de computação em gerar e armazenar dados é muito grande em relação à capacidade de análise destes dados na avaliação da qualidade de água (CHAU, 2006). Um sistema de informação pode ser consequentemente criado de modo a coletar, armazenar e compartilhar a informação além de auxiliar na tomada de decisão (SOUZA et al., 2010).

Assim, por exemplo, Liao e Sun (2010) estudaram a qualidade da água baseada em um modelo de árvore de decisão. Também, Siontorou e Batzias (2011) construíram uma ferramenta gerencial usando lógica Fuzzy aplicada à árvore de decisão; permitindo a incorporação de algum tipo de categorização a priori nas cadeias causais da estrutura hierarquizada. Em vista disso, como parte da proposta, pode ser construída uma árvore de decisão que é um diagrama que descreve as interações chaves entre as decisões e os eventos probabilísticos associados.

As árvores consistem de nós de decisão e incerteza que representam os atributos e os valores possíveis para estes atributos onde um problema complexo é decomposto em sub-problemas mais simples (REFFSTRUP et al., 2010; STEWART; PURUCKER, 2011). A árvore pode ser estruturada por dedução tanto de cima para baixo (envolvendo a identificação dos eventos ou série de eventos que contribuem diretamente para o evento de topo como presença de contaminação, as causas imediatas desses eventos e assim por diante, até que as causas finais sejam identificadas) quanto de baixo para cima (considerando os eventos iniciais, as respostas imediatas a esses eventos até que o evento final - risco de contaminação - seja determinado).

A árvore de decisão será então baseada principalmente em alguns critérios relativos às condições relacionadas às práticas de manejo e uso de agrotóxicos da área avaliada além dos dados obtidos para os indicadores quanto à exposição aos compostos presentes na mistura. Assim, para a inclusão dos indicadores bioquímicos selecionados, há necessidade de avaliação dos mesmos quanto ao seu nível de criticidade/importância, ou seja, a sequência na qual formam os nós da árvore de decisão de acordo com regras de classificação.

A criticidade (baixa, média e alta) pode ser estabelecida pela resposta dos diversos indicadores nas Fases II e III testados em pontos onde é potencialmente necessário prevenir, eliminar ou reduzir um perigo dentro de limites aceitáveis. Porém, para que as conclusões obtidas sejam representativas da realidade do local estudado, deve-se ter o maior cuidado possível na escolha dos critérios de desempenho dos indicadores, no processamento dos dados disponíveis, na seleção dos dados para alimentar o modelo e na sua arquitetura, na otimização e na validação do modelo (MAIER; DANDY, 2000). Portanto, a sequência de passos avaliados com a árvore de decisão dependerá do estado da arte da metodologia de avaliação de risco e das características de cada caso em estudo. 
A partir dos resultados é possível concluir que a capacidade para coletar e utilizar os dados obtidos para o processo decisório e para a gestão e formulação de políticas deve se refletir na ocorrência de efeitos de natureza: i.econômica - utilizar metodologias de menor custo, ii. social - aumentar a responsabilidade social e, iii. política - promover a melhoria de possíveis práticas deficientes. Nesse sentido, é importante uma política para a melhoria contínua dos laboratórios que fazem estes ensaios ecotoxicológicos visando a uma avaliação mais ampla e completa do impacto de agrotóxicos em diferentes níveis tróficos.

Os resultados obtidos com a abordagem proposta poderão fornecer dados ecotoxicológicos objetivos e atualmente escassos a respeito das descargas antropogênicas de origem agrícola no ambiente. A esse respeito, algumas enzimas como acetilcolinesterase têm sido investigadas quanto a sua capacidade de detecção de pesticidas em água e outras matrizes, tais como solo. Estas enzimas têm sido inclusive incorporadas a biossensores para esta finalidade (VAN DYK; PLETSCHKE, 2011).

Alguns estudos têm sido realizados para a determinação da atividade enzimática frente a diferentes concentrações de poluentes em organismos de diferentes níveis tróficos como microcrustáceos (JONSSON; AOYAMA, 2007; 2009) e peixes (JONSSON et al., 2002). Contudo, ainda faltam estudos em condições ambientais reais.

Desta forma, espera-se que as ações geradas pelos resultados obtidos dos indicadores biológicos sejam direcionadas a produção e compilação de um conjunto de dados por essa proposta que servirá para auxiliar a análise dos possíveis impactos ocasionados por esses produtos junto aos órgãos reguladores.

\section{Referências}

AMORIM, L. Os biomarcadores e sua aplicação na avaliação da exposição aos agentes químicos ambientais. Revista Brasileira de Epidemiologia, São Paulo, v. 6, Supl. 1, p. 1-13, 2003.

ANAND, S. S.; MEHENDALE, H. M. Liver regeneration: a critical toxicodynamic response in predictive toxicology. Environmental Toxicology and Pharmacology, Amsterdam, v. 18, p. 149-160, 2004.
ANDERSEN, I. S.; VOIE, O. A.; FONNUM, F.; MARIUSSEN, E. Effects of methyl mercury in combination with polychlorinated biphenyls and brominated flame retardants on the uptake of glutamate in rat brain synaptosomes: A mathematical approach for the study of mixtures. Toxicological Sciences, Oxford, v. 112, n. 1, p. 175-184, 2009.

ANDERSEN, M.; DENNISON, J. E. Mechanistic approaches for mixture risk assessments - Present capabilities with simple mixtures and future directions. Environmental Toxicology and Pharmacology, Amsterdam, v. 16, p. 1-11, 2004.

ARAÚJO, A.; LIMA, J.; MOREIRA, J.; JACOB, S.; SOARES, M.; MONTEIRO, M.; AMARAL, A.; KUBOTA, A.; MEYER, A.; COSENZA, A.; NEVES, C.; MARKOWITZ, S. Exposição múltipla a agrotóxicos e efeitos à saúde: Estudo transversal em amostra de 102 trabalhadores rurais, Nova Friburgo, RJ. Ciência \& Saúde Coletiva, Rio de Janeiro, v. 12, n. 1, p. 115-130, 2007.

ARIAS, A. R. L.; BUSS, D. F.; ALBURQUERQUE, C.; INÁCIO, A. F.; FREIRE, M. M.; EGLER, M.; MUGNAI, R.; BAPTISTA, D. F. Utilização de bioindicadores na avaliação de impacto e no monitoramento da contaminação de rios e córregos por agrotóxicos. Ciência \& Saúde Coletiva, Rio de Janeiro, v. 12, n. 1, p. 61-72, 2007.

ARNOLD, S. F.; PRICE, P. S. Modeling mixtures resulting from concurrent exposures to multiple sources. Toxicology and Applied Pharmacology, Orlando, v. 223, p. 121-124, 2007.

ARNOT, J.; ARMITAGE, J. M.; MC CARTY, L. S.; WANIA, F.; COUSINS, I.; TOOSE-REID, L. Toward a consistent evaluative framework for POP risk characterization. Environmental Science \& Technology, Washington, v. 45, p. 97-103, 2011.

BAAS, J.; JAGER, T.; KOOIJMAN, S. A. L. M. A model to analyze effects of complex mixtures on survival. Ecotoxicology and Environmental Safety, New York, v. 72, p. 669-676, 2009.

BALDWIN, W. S.; ROLING, J. A. A concentration addition model for the activation of the constitutive androstane receptor by xenobiotic mixtures. Toxicological Sciences, Oxford, v. 107, n. 1, p. 93-105, 2009.

BARROS, H.; SEDIYAMA, T.; REIS, M.; CECON, P. Efeito da aplicação de fungicidas e da época de colheita na qualidade sanitária de sementes de soja. Acta Scientiarum Agronomy, Maringá, v. 27, n. 4, p. 639-645, 2005.

BELLAS, J. Prediction and assessment of mixture toxicity of compounds in antifouling paints using the sea-urchin embryolarval bioassay. Aquatic Toxicology, Amsterdam, v. 88, p. 308315, 2008.

CEDERGREEN, N.; CHRISTENSEN, A. M.; KAMPER, A.; KUDSK, P.; MATHIASSEN, S. K.; STREIBIG, J. C.; SØRENSEN, $\mathrm{H}$. A review of independent action compared to concentration addition as reference models for mixtures of compounds with different molecular target sites. Environmental Toxicology and Chemistry, Houston, v. 27, n. 7, p. 1621-1632, 2008.

CHAU, K. A review on integration of artificial intelligence into water quality modeling. Marine Pollution Bulletin, Amsterdan, v. 52, p. 726-733, 2006.

CHÈVRE, N.; MAILLARD, E.; LOEPFE, C.; BECKER-VAN SLOOTEN, K. Determination of water quality standards for chemical mixtures: Extension of a methodology developed for herbicides to a group of insecticides and a group of pharmaceuticals. 
Ecotoxicology and Environmental Safety, New York, v. 71, p. 740-748, 2008.

CORY-SLECHTA, D. Studying toxicants as single chemicals: does this strategy adequately identify neurotoxic risk? Neurotoxicology, New York, v. 26, p. 491-510, 2005.

COSTA, P. M.; CAEIRO S.; DINIZ, M. S.; LOBO, J.; MARTINS, M.; FERREIRA, A. M.; CAETANO, M.; VALE, C.; DELVALLS, T. A.; COSTA M. H. Biochemical endpoints on juvenile Solea senegalensis exposed to estuarine sediments: the effect of contaminant mixtures on metallothionein and CYP1A induction. Ecotoxicology, Knoxville, v. 18, p. 988-1000, 2009.

DENTON, D. L.; WHEELOCK, C. E.; MURRAY, S. A.; HAMMOCK, B. D.; HINTON, D. E. Joint acute toxicity of esfenvalerate and diazinon to larval fathead minnows (Pimephales promelas). Environmental Toxicology and Chemistry, Houston, v. 22, p. 336-341, 2003.

ECKERMAN, D.; GIMENES, L.; SOUZA, R.; GALVÃO, P.; SARCINELLI, P.; CHRISMAN, J. Age related effects of pesticide exposure on neurobehavioral performance of adolescent farm workers in Brazil. Neurotoxicology and Teratology, Oxford, v. 29, p. 164-175, 2007.

EL-MASRI, H. A. Experimental and mathematical modeling methods for the investigation of toxicological interactions. Toxicology and Applied Pharmacology, Orlando, v. 223, p. 148154, 2007.

FENET, H.; CASELLAS, C.; BONTOUX, J. Laboratory and fieldcaging studies on hepatic enzymatic activities in european eel and rainbow trout. Ecotoxicology and Environmental Safety, New York, v. 40, p. 137-143, 1998.

GOMEZ-EYLES, J.L.; SVENDSEN, C.; LISTER, L.; MARTIN, H.; HODSON, M.E.; SPURGEON, D. J. Measuring and modelling mixture toxicity of imidacloprid and thiacloprid on Caenorhabditis elegans and Eisenia fetida. Ecotoxicology and Environmental Safety, New York, v. 72, p. 71-79, 2009.

GREIG-SMITH, P. W. A European perspective on ecological risk assessment, illustrated by pesticide registration procedures in the United Kingdom. Environmental Toxicology and Chemistry, Houston, v. 11, p. 1673-1689, 1992.

GROTEN, J. P.; HEIJNE, W. H. M.; STIERUM, R. H.; FREIDIG, A. P.; FERON, V. J. Toxicology of chemical mixtures: A challenging quest along empirical sciences. Environmental Toxicology and Pharmacology, Amsterdam, v. 18, p.185-192, 2004.

GUTIÉRREZ, S.; FERNÁNDEZ, C.; ESCHER, B. I.; TARAZONA, J. V. A new hazard index of complex mixtures integrates bioconcentration and toxicity to refine the environmental risk assessment of effluents. Environment International, Elmsford, v. 34, p.773-781, 2008.

JIN-CLARK, Y.; LYDY, M. J.; ZHU, K. Y. Effects of atrazine and cyanazine on chlorpyrifos toxicity in Chironomus tentans (Diptera: Chironomidae). Environmental Toxicology and Chemistry, Houston, v. 21, p. 598-603, 2002.

JONSSON, C. M.; AOYAMA, H. In vitro effect of agriculture pollutants and their joint action on Pseudokirchneriella subcapitata acid phosphatase. Chemosphere, Elmsford, v. 69, p. 849-855, 2007.

JONSSON, C. M.; AOYAMA, H. Extraction, partial characterization and susceptibility to $\mathrm{Hg} 2+$ of acid phosphatase from the microalgae
Pseudokirchneriella subcapitata. Scientia Agrícola, Piracicaba, v. 66, p. 634-642, 2009

JONSSON, C. M.; FERRACINI, V. L.; PARAIBA, L. C.; RANGEL, M; AGUIAR, S. R. Alterações bioquímicas em pacus (Metynnis argenteus) expostos ao paclobutrazol. Scientia Agricola, Piracicaba, v. 59, p. 441-446, 2002.

LAMA, P. K. S.; GRAY, J. S. The use of biomarkers in environmental monitoring programmes. Marine Pollution Bulletin, Amsterdan, v. 46, n. 2, p. 182-186, 2003.

LIAO, H.; SUN, W. Forecasting and evaluating water quality of chao lake based on an improved decision tree method procedia. Environmental Sciences, New York, v. 2, p. 970-979, 2010.

LIESS, M.; SCHÄFER, R. B.; SCHRIEVER, C. A. The footprint of pesticide stress in communities - Species traits reveal community effects of toxicants. Science of the Total Environment, Amsterdam, v. 406, p. 484-490, 2008.

LINDE-ARIAS, A. R.; INÁCIO, A. F.; NOVO, LEONARDO, A.; ALBURQUERQUE, C.; MOREIRA, J. C. Multibiomarker approach in fish to assess the impact of pollution in a large Brazilian river, Paraiba do Sul. Environmental Pollution, Amherst, v. 156, p. 974-979, 2008.

LIRA, R.; CONTRERAS, L. M.; RITA, R. M.; URBINA, J. A. Mechanism of action of anti-proliferative lysophospholipid analogues against the protozoan parasite Trypanosoma cruzi: Potentiation in vitro activity by the sterol biosynthesis inhibitor ketoconazole. Journal of Antimicrobial Chemotherapy, Oxford, v. 47, p. 537-546, 2001.

LU, G. H.; WANG, C.; WANG, P. F.; CHEN, Z. Y. Joint toxicity evaluation and QSAR modeling of aromatic amines and phenols to bacteria. Bulletin of Environmental Contamination and Toxicology, New York, v. 83, n. 1, p. 8-14, 2009.

LUSHCHAK, V. I. Environmentally induced oxidative stress in aquatic animals. Aquatic Toxicology, Amsterdam, v. 101, p. 1330,2011

MAGALHÃES D. P.; FERRÃO FILHO, A. S. A ecotoxicologia como ferramenta no biomonitoramento de ecossistemas aquáticos. Oecologia Brasiliensis, Rio de Janeiro, v. 12, n. 3, p. 355-381, 2008.

MAIER, H. R.; DANDY, G. C. Neural networks for the prediction and forecasting of water resources variables: A review of modelling issues and applications. Environmental Modelling \& Software, Amsterdam, v. 15, p. 101-124, 2000.

MASON, A. M.; BORGERT, C. J.; BUS, J. S.; MUMTAZ, M. M.; SIMMONS, J. E.; SIPES I. G. Improving the scientific foundation for mixtures joint toxicity and risk assessment: Contributions from the SOT mixtures project - Introduction. Toxicology and Applied Pharmacology, Orlando, v. 223, p. 99-103, 2007.

MATTSSON, J. L. Mixtures in the real world: The importance of plant self-defense toxicants, mycotoxins, and the human diet. Toxicology and Applied Pharmacology, Orlando, v. 223, p. 125132, 2007.

MUMTAZ, M. M.; RUIZ, P.; DE ROSA, C. T. Toxicity assessment of unintentional exposure to multiple chemicals. Toxicology and Applied Pharmacology, Orlando, v. 223, p. 104-113, 2007.

NASCIMENTO, I. A.; PEREIRA, S. A.; LEITE, M. B. N. L. Biomarcadores como instrumentos preventivos de poluição. In: ZAGATTO, P. A., BERTOLETTI, E. (Ed.). Ecotoxicologia 
aquática - Princípios e aplicações. São Carlos: Editora Rima, 2006. p. 413-432.

NEEDHAM, L. L.; BARR, D. B.; CAUDILL, S. P.; PIRKLE, J. L.; TURNER, W. E.; OSTERLOH, J.; JONES, R. L.; SAMPSON, E. J. Concentrations of environmental chemicals associated with neurodevelopmental effects in US population. Neurotoxicology, New York, v. 26, p. 531-545, 2005.

NEUWOEHNER, J.; ZILBERMAN, T.; FENNER, K.; ESCHER, B. I. QSAR-analysis and mixture toxicity as diagnostic tools: Influence of degradation on the toxicity and mode of action of diuron in algae and daphnids. Aquatic Toxicology, Amsterdam, v. 97, p. 58-67, 2010.

PÉRY, A. R. R.; DESMOTS, S.; MOMBELLI, E. Substancetailored testing strategies in toxicology: An in silico methodology based on QSAR modeling of toxicological thresholds and Monte Carlo simulations of toxicological testing. Regulatory Toxicology and Pharmacology, San Diego, v. 56, p. 82-92, 2010.

POHL, H. R.; ABADIN, H. G. Chemical mixtures: Evaluation of risk for child-specific exposures in a multi-stressor environment. Toxicology and Applied Pharmacology, Orlando, v. 233, p. 116$125,2008$.

POSTHUMA, L.; EIJSACKERS, H. J. P.; KOELMANS, A. A.; VIJVER, M. G. Ecological effects of diffuse mixed pollution are site-specific and require higher-tier risk assessment to improve site management decisions: A discussion paper. Science of the Total Environment, Amsterdam, v. 406, p. 503-517, 2008.

POUNDS, J. G.; HAIDER, J.; CHEN, D. G.; MUMTAZ, M. Interactive toxicity of simple chemical mixtures of cadmium, mercury, methylmercury and trimethyltin: model-dependent responses. Environmental Toxicology and Pharmacology, Amsterdam, v. 18, p. 101-113, 2004.

RAMLJAK, S.; HACKENBERGER, B. K.; SMITAL, T.; BRITVIĆ, S. Evaluation of the genotoxic and cytochrome P450 monooxygenase-inhibitory potential of dicuran on prokaryotic and eukaryotic test systems. Journal of Environmental Science and Health B, New York, v. 35, n. 6, p. 751-770, 2000.

REFFSTRUP, T. K.; LARSEN, J. C.; MEYER, O. Risk assessment of mixtures of pesticides. Current approaches and future strategies. Regulatory Toxicology and Pharmacology, San Diego, v. 56, n. 2, p. 174-192, 2010.

REIS FILHO, E.; ARDUIM, G.; BARCELLOS, A.; MOREIRA, E. Sobre a alteração de sensibilidade de raças de Puccinia triticina, agente causal da ferrugem da folha do trigo, a alguns fungicidas. Revista Plantio Direto, Passo Fundo, v.100, p. 15-17, 2007.

REISFELD, B.; YANG, R. S. H. A reaction network model for CYP2E1-mediated metabolism of toxicant mixtures. Environmental Toxicology and Pharmacology, Amsterdam, v. 18, p. 173-179, 2004.

RICE, G.; MACDONELL, M.; HERTZBERG, R. C.; TEUSCHLER, L.; PICEL, K.; BUTLER, J.; CHANG, Y.; HARTMANN, H. An approach for assessing human exposures to chemical mixtures in the environment. Toxicology and Applied Pharmacology, Orlando, v. 233, p. 126-136, 2008.

RUUS, A.; SANDVIK, M.; UGLAND, K. I.; SKAARE, J. U. Factors influencing activities of biotransformation enzymes, concentrations and compositional patterns of organochlorine contaminats in members of a marine food web. Aquatic Toxicology, Amsterdam, v. 61, p. $73-87,2002$.

SEXTON, K.; HATTIS, D. Assessing cumulative health risks from exposure to environmental mixtures-three fundamental questions. Environmental Health Perspectives, Weston Parkway Cary, v. 115 , n. 5, p. 825-832, 2007.

SIONTOROU, C. G.; BATZIAS, F. A. Error identification/ propagation/remediation in biomonitoring survey - A knowledgebased approach towards standardization via fault tree analysis. Ecological Indicators, Amsterdam, v. 11, p. 564-581, 2011.

SMIALOWICZ, R. J.; DEVITO, M. J.; WILliAMS, W. C.; BIRNBAUM, L. S. Relative potency based on hepatic enzyme induction predicts immunosuppressive effects of a mixture of PCDDS/PCDFS and PCBS. Toxicology and Applied Pharmacology, Orlando, v. 227, p. 477-484, 2008.

SOUZA, Z. M.; CERRI, D. G. P.; COLET, M. J.; RODRIGUES, L. H. A.; MAGALHÃES, P. S. G.; MANDONI, R. J. A. Análise dos atributos do solo e da produtividade da cultura de cana-de-açúcar com o uso da geoestatística e árvore de decisão. Ciência Rural, Santa Maria, v. 40, n. 4, p. 840-847, 2010.

SPURGEON, D. J.; JONES, O. A. H.; DORNE, J.-L. C. M.; SVENDSEN, C.; SWAIN, S.; STÜRZENBAUM, S. R. Systems toxicology approaches for understanding the joint effects of environmental chemical mixtures. Science of the Total Environment, Amsterdam, v. 408, p. 3725-3734, 2010.

STEWART, R. N.; PURUCKER, S. T. An environmental decision support system for spatial assessment and selective remediation. Environmental Modelling \& Software, Amsterdam, v. 26, p.751$760,2011$.

SUTER II, G. W.; CORMIER, S. M. Why and how to combine evidence in environmental assessments: Weighing evidence and building cases. Science of the Total Environment, Amsterdam, v. 409, p. 1406-1417, 2011.

TARJA, N.; KIRSTI, E.; MARJA, L.; KARI, E. Thermal and metabolic factors affecting bioaccumulation of triazine herbicides by rainbow trout (Oncorhynchus mykiss). Environmental Toxicology, New York, v. 18, n. 4, p. 219-226, 2003.

TAYSSE, L.; CHAMBRAS, C.; MARIONNET, D.; BOSGIRAUD, C.; DESCHAUUX, P. Basal level and induction of cytochrome P450, EROD, UDPGT, and GST activities in carp (Cyprinus carpio) immune organs (spleen and head kidney). Bulletin of Environmental Contamination and Toxicology, New York, v. 60, p. $300-305,1998$.

TEUSCHLER, L. K. Deciding which chemical mixtures risk assessment methods work best for what mixtures. Toxicology and Applied Pharmacology, Orlando, v. 223, p. 139-147, 2007.

TOYOSHIBA, H.; WALKER, N. J.; BAILER, A. J.; PORTIER, C. J. Evaluation of toxic equivalency factors for induction of cytochromes P450 CYP1A1 and CYP1A2 enzyme activity by dioxin-like compounds. Toxicology and Applied Pharmacology, Orlando, v. 194, p. 156-168, 2004.

USEPA. Guidance on cumulative risk assessment of pesticide chemicals that have a common mechanism of toxicity. Washington: Office of pesticide programs. US environmental protection agency, 2002. $81 \mathrm{p}$.

VAN DER OOST, R.; BEYER, J.; VERMEULEN, N. Fish bioaccumulation and biomarkers in environmental risk assessment: 
A review. Environmental Toxicology and Pharmacology, Amsterdam, v. 13, p. 57-149, 2003.

VAN DYK, J. S.; PLETSCHKE, B. Review on the use of enzymes for the detection of organochlorine, organophosphate and carbamate pesticides in the environment. Chemosphere, Elmsford, v. 82, p. 291-307, 2011.

WANG, C.; FOWLER, B. A. Roles of biomarkers in evaluating interactions among mixtures of lead, cadmium and arsenic. Toxicology and Applied Pharmacology, Orlando, v. 233, p. 9299, 2008.

WANG, C.; LU, G.; TANG, Z.; GUO, X. Quantitative structureactivity relationships for joint toxicity of substituted phenols and anilines to Scenedesmus obliquus. Journal of Environmental Science, New York, v. 20, n. 1, p. 115-119, 2008.
WHO - WORLD HEALTH ORGANIZATION. Assessment of combined exposures to multiple chemicals: report of a WHO/IPCS international workshop on aggregate/cumulative risk assessment. (IPCS Harmonization Project Document n. 7). Geneva: World Health Organization, 2009. 75 p.

WU, H. C.; CHEN, P. C.; TSAY, T. T. Assessment of nematode community structure as a bioindicator in river monitoring. Environmental Pollution, Amherst, v. 158, p. 1741-1747, 2010.

ZHANG, 1.; ZHOU, P. J.; YANG, F.; WANG, Z. D. Computerbased QSARs for predicting mixture toxicity of benzene and its derivatives. Chemosphere, Elmsford, v. 67, n. 2, p. 396-401, 2007. 